\title{
Usefulness of serum protein profiling for prediction of preoperative chemoradiosensitivity of esophageal cancer
}

\author{
YOSHIHIRO OTA ${ }^{1}$, YU TAKAGI ${ }^{1}$, YOSHIAKI OSAKA ${ }^{1}$, MOTOO SHINOHARA ${ }^{1}$, SUMITO HOSHINO ${ }^{1}$, \\ AKIHIKO TSUCHIDA ${ }^{1}$, TATSUYA AOKI ${ }^{1}$, KAZUFUMI HONDA ${ }^{2}$ and TESSHI YAMADA ${ }^{2}$ \\ ${ }^{1}$ Third Department of Surgery, Tokyo Medical University; ${ }^{2}$ Chemotherapy Division and Cancer \\ Proteomics Project, National Cancer Center Research Institute, Tokyo 160-0023, Japan
}

Received March 2, 2007; Accepted May 23, 2007

\begin{abstract}
We examined whether serum protein profiling is a reliable index for prediction of therapeutic efficacy of preoperative chemoradiotherapy (PCRT) in advanced esophageal cancer compared with evaluation of the efficacy of conventional clinical examination. We entered 42 patients who received PCRT and surgery between 1998 and 2002 into this study. Serum protein profiling was performed using the preoperative serum of the patient to select the marker set that enabled the efficacy of PCRT to be evaluated accurately. The efficacy of PCRT was predicted with the marker set, and the sensitivity, specificity and accuracy of the method were calculated based on evaluation of the efficacy by pathological examination. Similarly, therapeutic efficacy was also predicted based on evaluation of the efficacy of conventional clinical examination, and the results were compared with those of prediction by serum protein profiling. The correlation between each predictive examination and outcome was evaluated. The sensitivity, specificity and accuracy of prediction of therapeutic efficacy of PCRT by serum protein profiling were $90.9,100$ and $93.3 \%$, respectively. In clinical examination, prediction of the efficacy of PCRT by three methods was as follows: by esophagography, sensitivity $76.0 \%$, specificity $17.6 \%$, accuracy $52.4 \%$; by endoscopy, sensitivity $80.0 \%$, specificity $11.8 \%$, accuracy $52.4 \%$; by computed tomography, sensitivity $60.0 \%$, specificity $47.1 \%$, accuracy $54.8 \%$, respectively. These results demonstrated the superiority of serum protein profiling in predicting the therapeutic efficacy of PCRT compared with conventional clinical examination. Moreover, serum protein profiling was the only significant prognostic factor as regards the correlation with outcome by multivariate analysis.
\end{abstract}

Correspondence to: Dr Akihiko Tsuchida, Third Department of Surgery, Tokyo Medical University, 6-7-1 Nishi-Shinjuku, Shinjukuku, Tokyo 160-0023, Japan

E-mail: akibobo@hotmail.com

Key words: esophageal cancer, chemoradiotherapy, serum protein profiling, proteomics

\section{Introduction}

Esophageal cancer generally has a poor prognosis and cannot be cured by surgery alone because it readily develops lymph node metastasis or invades into the trachea, bronchi or large vessels, especially if advanced. Since esophageal cancer is sensitive to chemotherapy and radiotherapy, various approaches to multidisciplinary treatment in which chemoradiotherapy is combined with surgical treatment have been made. Among them, preoperative chemoradiotherapy (PCRT), which came into use in the 1980s in Europe and North America, is reported to enhance the treatment results of advanced esophageal cancer by improving resection rates evaluating local control or by inhibiting post-operative recurrence by controlling minute metastatic lesions (1-3). However, several studies have revealed that PCRT does not affect all patients with esophageal cancer but improves the survival rates only when it is pathologically effective $(4,5)$. In ineffective cases, PCRT is actually disadvantageous due to its side effects. It is therefore important to properly predict the therapeutic efficacy of PCRT and to use PCRT only for those cases in which effect can be anticipated. In order to discover a new diagnostic that can accurately predict the efficacy of PCRT, we performed serum protein profiling using preoperative serum of the patient and reported that efficacy could be accurately predicted (6).

In the present study, we compared the evaluation of efficacy of conventional clinical examination as regards the accuracy of prediction and correlation with outcome, and objectively assessed the prediction of therapeutic efficacy of PCRT by serum protein profiling to gain insight into the usefulness of PCRT.

\section{Patients and methods}

Patients. Forty-two patients with esophageal squamous cancer that underwent PCRT and surgery at the Third Department of Surgery, Tokyo Medical University Hospital between January 1998 and December 2002 were enrolled in the study. The characteristics of the patients were as follows: mean age, 61.7; gender, 37 men and 5 women; tumor location, 2 in cervical area; 7 in the upper thoracic area; 21 in the middle thoracic area; 11 in the lower thoracic area; and 1 
Table I. Patient characteristics.

\begin{tabular}{lr}
\hline No. of patients & 42 \\
Male/female & $37 / 5$ \\
Age (years, mean \pm SD) & $61.7 \pm 6.9$ \\
Tumor location & \\
Ce & 2 \\
Ut & 7 \\
Mt & 21 \\
Lt & 11 \\
Ae & 1 \\
Clinical stage & \\
II & 7 \\
III & 29 \\
IV & 6
\end{tabular}

$\overline{\mathrm{Ce}}$, cervical esophagus; Ut, upper thoracic esophagus; $\mathrm{Mt}$, middle thoracic esophagus; Lt, lower thoracic esophagus; Ae, abdominal esophagus.

in abdomen; clinical stage, 7 in stage II; 29 in stage III; and 6 in stage IV (Table I).

Preoperative chemoradiotherapy (PCRT). In PCRT, low dose FP (CDDP $10 \mathrm{mg} / \mathrm{m}^{2} /$ day, 5 days a week for 2 weeks, total $100 \mathrm{mg} / \mathrm{m}^{2}+5$-FU $350 \mathrm{mg} / \mathrm{m}^{2} /$ day, 5 days a week for 4 weeks, total $\left.7,000 \mathrm{mg} / \mathrm{m}^{2}\right)$ and radiation $(10-\mathrm{MV}$ linear accelerator 2 Gy/day, 5 days a week for 4 weeks, total 40 Gy) were administered concurrently. The radiation field was set to include both the primary lesion and accessory lymph nodes. Approximately 2 weeks after PCRT, the effects on the primary lesion and accessory lymph nodes were assessed by esophagography, esophagoscopy, computed tomography (CT) and endoscopic ultrasonography. The operation was performed $\sim 4$ weeks after PCRT. The esophagus was resected via a right thoracotomy, and 3-field lymphadenectomy was performed in the cervical, thoracic and abdominal regions.

Radiological and pathological criteria for the effects of $P C R T$. The criteria for radiological response in the primary lesion were defined by the Japanese Society for Esophageal Disease (7). Complete response (CR), the disappearance of tumor shadow on esophagography plus disappearance of the tumor and a flat mucosal surface on esophagography; partial response $(\mathrm{PR})$, a reduction rate $[\mathrm{RR}=$ (pretreatment tumor volume)-(post-treatment tumor volume)/(pretreatment tumor volume $\mathrm{x} 100 \%$ )] of $50 \%$ or more, on esophagography, there is a marked reduction in tumor shadow, and on esophagography, there is a flattening of tumor or reduction of protrusions at the periphery of the ulcer, plus decreased depth of the ulcer; no change (NC), RR is $<50 \%$ or increases by up to $25 \%$, on esophagography, there is either no change or a slight reduction of the tumor and no change or flattening of the protrusion at the ulcer periphery; progressive disease (PD), RR increases $25 \%$ or more, on esophagography, there is an increase of the tumor shadow or the appearance of a new lesion. In evaluating the therapeutic efficacy of PCRT, CR and PR were evaluated as effective and $\mathrm{NC}$ and PD as ineffective.

Pathological response criteria were defined as follows: grade 0 , no discernible therapeutic effect on cancer tissue or cells; grade 1 , apparently viable cells account for $1 / 3$ or more of tumor tissue, but there is some evidence of degeneration of cancer tissue or cells; grade 2, viable cancer cells account for $<1 / 3$ of tumor tissue, while other cancer cells are severely degenerated or necrotic; grade 3 , no viable cancer cells evident. Grades 0 and 1 were evaluated as ineffective and grades 2 and 3 as effective.

Surface-enhanced laser desorption and ionization coupled with hybrid quadrupole time-of-flight mass spectrometry (SELDI-TOF-MS) analysis. To denature serum proteins, $90 \mu 1$ of U9 buffer $\{9 \mathrm{~mol} / \mathrm{l}$ urea, $2 \% 3$-[(3-cholamidopropyl) dimethylammonio]-1-propanesulfonic acid, and $50 \mathrm{mmol} / \mathrm{l}$ Tris-HCl (pH 9)\} was added to $10 \mu \mathrm{l}$ of each sample and vortexed for $20 \mathrm{~min}$. To increase the number of detectable protein peaks, we used 4 different ProteinChip (Ciphergen Biosystems, Inc., Fremont, CA, USA) array/wash conditions, i.e., reversed phase (H50), weak cation exchanger with low stringent wash $(\mathrm{CM} 10 / \mathrm{pH} 4.0)$, cation exchanger with high stringent wash (CM10/pH 7.0) and immobilized metal affinity capture coupled with copper (IMAC-Cu ${ }^{2+}$ ), as instructed by the supplier. Each sample was randomly assigned in duplicate to one of 96 spots of 12 allied ProteinChip arrays with a Biomek 2000 laboratory workstation (Beckman Coulter Inc., Fullerton, CA, USA). Sinapinic acid solution was prepared in $50 \% \mathrm{v} / \mathrm{v}$ acetonitrile and $5 \% \mathrm{v} / \mathrm{v}$ trifluoroacetic acid as an energy-absorbing matrix, and $1 \mu 1$ of saturated solution was applied to each spot on the chips. Low-molecular-weight proteins in the $2,000-40,000 \mathrm{~m} / \mathrm{z}$ range were read on a highresolution performance hybrid quadrupole time-of-flight mass spectrometer Q-star XL (Applied Biosystems, Foster City, CA) equipped with a PCI 1000 ion source (Ciphergen). The laser intensity, frequency, and accumulation time of the instrument were set at $60 \%, 25 \mathrm{~Hz}$, and $90 \mathrm{sec}$, respectively. Mass accuracy was externally calibrated on the day of the measurements by using the all-in-one-peptide molecular mass standard (Ciphergen).

Peak detection and quantification. The mass data were converted to text files consisting of $\mathrm{m} / \mathrm{z}$ and intensity after mass calibration by Analyst QS (Applied Biosystems) and processed by the following procedures. First, the spectrum was smoothed with a Gaussian window function (weighting addition by the following function). Baseline and noise level were calculated by making the bottom 10 percentile in descending order and $2 \times$ (10-5\% point), respectively, for every $200 \mathrm{~m} / \mathrm{z}$ interval, which divided the range of $2,000-40,000 \mathrm{~m} / \mathrm{z}$, and by connecting each section by natural spline interpolation (8). Maximum intensity was calculated for every $1 \mathrm{~m} / \mathrm{z}$ interval. All apices and valleys of the intensity arrangement were detected first. The depths of adjoining low peaks and valleys were then calculated, and if the depth was $<4$ signal-to-noise ratios $(\mathrm{S} / \mathrm{N})$, it was merged into higher peaks. Peak alignment was performed so that the number of samples with a $>8 \mathrm{~S} / \mathrm{N}$ peak in the tolerance level of $\mathrm{m} / \mathrm{z} 0.05 \%$ would become the maximum. If a sample had a $>16 \mathrm{~S} / \mathrm{N}$ peak or the proportion 


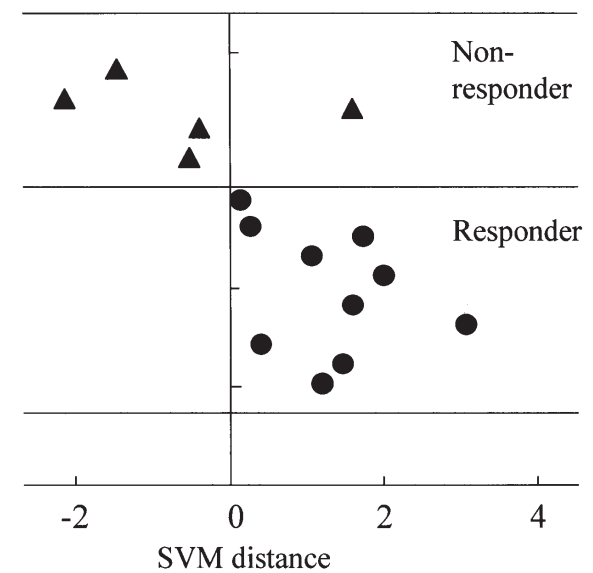

Figure 1. Results of serum protein profiling in the validation set $(n=15)$. Sensitivity is $90.9 \%(10 / 11)$, specificity is $100 \%(4 / 4)$ and accuracy is $93.3 \%(14 / 15)$.

of samples with a $>4 \mathrm{~S} / \mathrm{N}$ peak was $>40 \%$, the peaks were used for marker discovery. Finally, peak intensity was normalized so that the total intensity after baseline subtraction would be the same for all samples in the 2,000$40,000 \mathrm{~m} / \mathrm{z}$ range. Peak images were generated with Analyst QS and MassNavigator software (Mitsui Knowledge Industry, Tokyo, Japan).

SELDI-TOF-MS was used to perform serum protein profiling. Serum of a total of 27 patients, 15 responders of grade 2 and 3 and 12 non-responders of grade 0 and 1 with no significant difference in age, gender, tumor location and clinical stage, underwent serum protein profiling as a training set, and the profiling pattern that could distinguish responders and non-responders with an accuracy of $100 \%$ was identified and a marker set was extracted. Usefulness of the marker set was then examined with a validation set consisting of 15 other cases (10 responders and 5 non-responders).

Evaluation of PCRT and statistical analysis. The therapeutic efficacy of PCRT was examined by evaluating the efficacy of conventional clinical examinations and by serum protein profiling to examine the sensitivity, specificity and accuracy of individual items by comparison with the efficacy of pathological response. In addition, cumulative survival rates of the 42 patients were calculated by the Kaplan-Meier method, and the correlation between outcome and the prediction of the therapeutic efficacy based on evaluation of clinical examination and from serum protein profiling was examined using univariate and multivariate analyses. A p-value of $<0.05$ was considered to indicate a statistically significant difference.

\section{Results}

Predictive efficacy of serum protein profiling. Serum protein profiling was performed on 42 patients with esophageal cancer using SELDI-TOF-MS and a total of 859 peak values (mean correlation coefficient: $0.960 \pm 0.019)$ that reflected serum samples within the range of 2,000-40,000 m/z were selected. From those peak values, the combination of peaks that enabled the best predictive efficacy was sought using the training set of 27 case specimens with the support vector machine (SVM). As a result, the combination of four peaks of 7,420 (H50), 9,112 (H50), 17,123 (CM10/pH 4.0) and 12,876 (IMAC$\left.\mathrm{Cu}^{2+}\right) \mathrm{m} / \mathrm{z}$ was found as a biomarker to be able to diagnose the 15 responders with $100 \%$ sensitivity and the 12 nonresponders with $100 \%$ specificity. These biomarkers were then evaluated using a validation set of 15 cases. The result was $90.9 \%$ sensitivity (10/11), $100 \%$ specificity (4/4) and $93.3 \%$ accuracy $(14 / 15)$, demonstrating that serum protein profiling provided highly accurate prediction of the therapeutic efficacy of PCRT (Fig. 1).

Relationship between the predictive efficacy of conventional clinical examination and pathological response. The predictive efficacies of conventional clinical examination and pathological response were compared. The sensitivity, specificity and accuracy of the efficacy evaluation were as follows respectively: by esophagography, $76.0 \%(19 / 25), 17.6 \%(3 / 17)$ and $52.4 \%(22 / 42)$; by endoscopy, $80.0 \%(20 / 25), 11.8 \%$ $(2 / 17)$ and $52.4 \%(22 / 42)$; by CT, $60.0 \%(15 / 25), 47.1 \%$ $(8 / 17)$ and $54.8 \%(23 / 42)$ (Table II). These figures showed

Table II. Relationship between the predictive efficacy of conventional examination and pathological response in primary lesion.

\begin{tabular}{|c|c|c|c|c|c|c|}
\hline & \multicolumn{2}{|c|}{ Esophagography } & \multicolumn{2}{|c|}{ Endoscopy } & \multicolumn{2}{|c|}{$\mathrm{CT}$} \\
\hline & $\mathrm{CR}+\mathrm{PR}$ & $\mathrm{NC}$ & $\mathrm{CR}+\mathrm{PR}$ & $\mathrm{NC}$ & $\mathrm{CR}+\mathrm{PR}$ & $\mathrm{NC}$ \\
\hline \multicolumn{7}{|c|}{ Pathological response } \\
\hline Grade 2 and 3 & 19 & 6 & 20 & 5 & 15 & 10 \\
\hline Grade 0 and 1 & 14 & 3 & 15 & 2 & 9 & 8 \\
\hline Sensitivity (\%) & \multicolumn{2}{|c|}{76.0} & \multicolumn{2}{|c|}{80.0} & \multicolumn{2}{|c|}{60.0} \\
\hline Specificity $(\%)$ & \multicolumn{2}{|c|}{17.6} & \multicolumn{2}{|c|}{11.8} & \multicolumn{2}{|c|}{47.1} \\
\hline Accuracy $(\%)$ & \multicolumn{2}{|c|}{52.4} & \multicolumn{2}{|c|}{52.4} & \multicolumn{2}{|c|}{54.8} \\
\hline
\end{tabular}

$\mathrm{CR}$, complete response; $\mathrm{PR}$, partial response; $\mathrm{NC}$, no change. 
Table III. Univariate and multivariate analysis in each predictive efficacy for survival.

\begin{tabular}{lccccc}
\hline & & \multicolumn{2}{c}{ Confidence interval } & & Univariate \\
Variable & Hazard ratio & $<95 \%$ & $>95 \%$ & & $\begin{array}{c}\text { Multivariate } \\
\text { p-value }\end{array}$ \\
\hline Esophagography & 0.851 & 0.282 & 2.571 & 0.7749 & 0.7028 \\
Endoscopy & 1.266 & 0.419 & 3.827 & 0.5150 & 0.2752 \\
CT & 0.738 & 0.296 & 1.840 & 0.6756 & 0.3435 \\
Proteome & 2.896 & 1.154 & 7.267 & 0.0234 & 0.0144 \\
Pathology & 2.522 & 1.018 & 6.252 & 0.0457 & 0.0254 \\
\hline
\end{tabular}

that prediction by conventional clinical examination is less accurate than by serum protein profiling.

Correlation between each predictive examination and survival. The correlation between each predictive examination method and survival was evaluated by univariate and multivariate analyses. Prediction by serum protein profiling proved to be the only effective prognostic factor, with $\mathrm{p}=0.0234$ on univariate analysis and $\mathrm{p}=0.0144$ on multivariate analysis (Table III). In addition, pathological response was also an effective prognostic factor with $\mathrm{p}=0.0457$ in the univariate analysis and $\mathrm{p}=0.0254$ in the multivariate analysis in agreement with previous reports.

\section{Discussion}

We set out to determine whether serum protein profiling is useful for predicting therapeutic efficacy of PRCT. Recent significant technological innovations in mass analysis in basic studies of proteomics have allowed highly sensitive detection of protein from small amounts of samples (9). Research and development have therefore been carried out to find new biomarkers using serum protein profiling. Many groups of patients and control subjects have been prepared as training sets whose age, gender, timing of blood sampling, method of blood sampling and blood preservation method are matched, and various machine learning algorithms (support vector machine) that can be applied to artificial intelligence have been made to study the proteomic patterns of both groups and hence extract patient-specific data sets. Once the data sets are precisely defined, both groups can be accurately distinguished even when validation samples without any clinical information are examined (10). We have so far performed serum protein profiling on preoperative serum of patients with esophageal cancer using SELDI-TOF-MS and reported that both responders and non-responders of PCRT could be predicted accurately (6). In this study, the usefulness of prediction by serum protein profiling was validated by making a comparison with the evaluation of the efficacy of conventional clinical examination by esophagography, endoscopy and CT. These results showed the superiority of serum protein profiling for predicting efficacy in terms of sensitivity, specificity and accuracy compared with conventional clinical examinations. One of the reported reasons for this is a probable inconsistency between evaluation of the efficacy of conventional clinical examinations and pathological response (11). In the present study, $42.4 \%$ (14/33), $42.9 \%(15 / 35)$ and $37.5 \%(9 / 24)$ of cases respectively classified as effective on the basis of esophagography, endoscopy and CT were found to be pathologically grade 0 and 1 . This indicates an inconsistency between efficacy evaluation by diagnostic imaging and by histopathological examination. This is attributable to at least the following two reasons: i) there are cases in which viable cancer cells remain in large numbers, despite seemingly decreasing tumor volumes on clinical examinations; ii) viable cancer cells are not easily detected in endoscopic biopsy, because resected specimens show that they are mainly scattered from submucosal layers to muscle layers, epidermis of which is usually covered with regenerative epithelia. Shiozaki et al reported similar results $(12,13)$. Predicting pathological response from diagnostic imaging alone, such as esophagography, endoscopy or CT, is extremely difficult. Although PCRT is advantageous in significantly effective cases, in ineffective cases one whole month might pass in vain, and the best timing for operation could be lost. It is therefore important to predict the therapeutic efficacy of PCRT before treatment and perform chemoradiation only in those cases in which it is likely to be effective. Our results suggest that serum protein profiling is the most accurate method available today.

Apart from esophagography, endoscopy and CT, endoscopic ultrasonography is reported to be useful for evaluating efficacy for clinical examination (14), although it is not normally used due to problems related to manipulative skills and evaluation criteria. Positron emission tomography (PET) is also reported to be useful, and has been gradually recognized as a less-invasive examination technique that can directly reflect pathological response $(15,16)$, although it has not yet been compared with pathological response because of a lack of general availability due to difficulties of the facilities and cost. Moreover, although it is still at the investigative stage, expression of mutant p53 protein in tumors and chemoradiosensitivity has been reported. Lowe et al (17) reported that after chemoradiation was performed on nude mice implanted subcutaneously with embryonic fibroblasts with or without p53 genes, a high proportion of tumors with p53 genes 
displayed apoptosis and their development was inhibited. The same report described extensive mutation when exons 5-8 of those tumors with p53 genes that did not respond to the treatment were observed, suggesting that the expression of wild-type p53 protein in a tumor can be a marker for chemoradiosensitivity. On the basis of this report, Ikeguchi et al (18) examined the expression of mutant p53 protein and the duration of cancer recurrence on resected specimens of a progressive esophageal cancer case that was categorized as non-curative resection due to residual cancer cells, and reported the potential of the mutant p53 protein expression as a marker for chemoradiosensitivity (19). Unfortunately, the usefulness of the expression of mutant $\mathrm{p} 53$ protein as a marker for sensitivity to PCRT has not yet been established. Apart from $\mathrm{p} 53$, several studies have reported $\mathrm{p} 21$ and other genetic markers and efficacy prediction factors such as CDC25B, VEGF and CD34 (20,21), but clinical applications of these results are limited and not yet established. Most studies on the malignancy of cancer by molecular biological approaches constitute retrospective examinations. If those studies are to be applied to clinical diagnoses, prospective studies must be conducted, but such studies require biopsy specimens and so only materials from part of the tumor surface are used for the evaluation; it is questionable whether such materials represent the whole characteristics of a tumor. All cancer cells in a tumor are rarely stained uniformly when the entire tumor is stained; in most cases stained and non-stained parts coexist, so false positives or false negatives can easily occur when the evaluation is based on immunostaining of biopsy specimens. In contrast, serum protein profiling can obtain virtually the same results regardless of age, gender, tumor location and clinical stage as well as sampling method, sampling volume and other conditions of the tumor.

Pathological response in resected specimens has been shown to correlate with prognosis, and a good prognosis is expected in cases where PCRT is pathologically effective. Serum protein profiling was shown in the present study to be an independent prognostic factor in univariate and multivariate analyses of the correlation with outcome, as pathological response can be accurately predicted by proteomic profiling. All cases shown to be sensitive to PCRT by serum protein profiling can therefore candidate for preoperative treatment (22). Although further studies with more samples are needed for clinical applications, prediction of the therapeutic efficacy of PCRT in esophageal cancer by serum protein profiling using the patient's serum is useful for application of PCRT and prediction of prognosis, promoting patient-specific treatment for individual patients with esophageal cancer.

\section{Acknowledgements}

This study was supported by the 'Third-Term Comprehensive Control Research for Cancer' conducted by the Ministry of Health, Labor and Welfare of Japan. The authors are indebted to Professor J. Patrick Barron of the International Medical Communications Center of Tokyo Medical University for his review of this manuscript.

\section{References}

1. Le Prise E, Etienne PL, Meunier B, et al: A randomized study of chemotherapy, radiation therapy, and surgery versus surgery for localized squamous cell carcinoma of the esophagus. Cancer 73: 1779-1784, 1994.

2. Apinop C, Puttisak P and Preecha N: A prospective study of combined therapy in esophageal cancer. Hepatogastroenterol 41: 391-393, 1994.

3. Bosset JF, Gignoux M, Triboulet JP, et al: Chemoradiotherapy followed by surgery compared with surgery alone in squamouscell cancer of the esophagus. N Engl J Med 337: 161-167, 1997.

4. Osaka Y, Takagi Y, Tsuchida A, et al: Concurrent preoperative chemoradiotherapy for stage III or IV esophageal squamous carcinoma. Oncol Rep 12: 1121-1126, 2004.

5. Osaka Y, Takagi Y, Hoshino S, et al: Effective of preoperative chemoradiotherapy for advanced esophageal carcinoma. J Jap Coll Surg 29: 6-12, 2004.

6. Hayashida Y, Honda K, Osaka Y, et al: Possible prediction of chemoradiosensitivity of esophageal cancer by serum protein profiling. Clin Cancer Res 11: 8042-8047, 2005.

7. The Japanese Society for Esophageal Diseases: Guidelines for the Clinical and Pathologic Studies on Carcinoma of the Esophagus. Isono K (ed). 9th edition. Kanehara Shuppan, Tokyo, 1999.

8. Gras R, Muller M, Gasteiger E, et al: Improving protein identification from peptide mass fingerprinting through a parameterized multi-level scoring algorithm and an optimized peak detection. Electrophoresis 20: 3535-3550, 1999.

9. Yamada T: Proteome-based approach to cancer diagnosis and treatment. J Jap Soc Oral Maxillofacial Surg 51: 8-9, 2005.

10. Aoshima K: The proteomics general remarks. Cognition Dementia 3: 217-231, 2004.

11. Doki Y, Kabuto T, Ishikawa O, et al: Diagnostic image for neoadjuvant chemoradiation therapies toward advanced esophageal cancers. Surg Treat 84: 454-464, 2001.

12. Shiozaki $\mathrm{H}$ and Yano M: Neoadjuvant chemoradiotherapy in patients with surgically treated T4 esophageal squamous cell carcinoma. J Jap Surg Soc 103: 284-289, 2002.

13. Yano M, Inoue M and Shiozaki H: Preoperative concurrent chemotherapy and radiation therapy followed by surgery for esophageal cancer. Ann Thorac Cardiovasc Surg 8: 123-130, 2002.

14. Zuccaro G Jr, Rice TW, Goldblum J, et al: Endoscopic ultrasound cannot determine suitability for esophagectomy after aggressive chemoradiotherapy for esophageal cancer. Am J Gastroenterol 94: 906-912, 1999.

15. Kato H, Kuwano H, Nakajima M, et al: Usefulness of positron emission tomography for assessing the response of neoadjuvant chemoradiotherapy in patients with esophageal cancer. Am J Surg 184: 279-283, 2002.

16. Brucher BL, Weber W, Bauer M, et al: Neoadjuvant therapy of esophageal squamous cell carcinoma; response evaluation by positron emission tomography. Ann Surg 233: 300-309, 2001.

17. Lowe SW, Bodis S, McClatchey A, et al: P53 status and the efficacy of cancer therapy in vivo. Science 266: 807-810, 1991.

18. Ikeguchi $M$ and Maeta $M$ : The expression of mutated $\mathrm{p} 53$ protein and proliferative activity of cancer cells in patients with esophageal squamous cell carcinoma. J Yonago Med Ass 49: 261-267, 1998.

19. Ikeguchi M, Sato H, Kitano K, et al: Radiochemosensitivity and expression of p53 in patients with esophageal cancer treated by avsolute non-curative resection. Anticancer Res 18: 493-498, 1998.

20. Kishi K, Doki Y, Miyata H, et al: Prediction of the response to chemoradiation and prognosis in oesophageal squamous cancer. Br J Surg 89: 597-603, 2002.

21. Imdahl A, Borgnar G, Schulte-Monting J, et al: Predictive factors for response to neoadjuvant therapy in patients with esophageal cancer. Euro J Card-thorac Surg 21: 657-663, 2002 .

22. Okumura H, Natsugoe S, Yokomakura N, et al: The new criteria of clinical response for the primary tumor based on the findings of histological response after chemoradiation therapy in esophageal cancer. Jpn J Gastroenterol Surg 38: 1637-1644, 2005. 